\title{
Study of Solution of Energy for a Nonlocal Semilinear Parabolic Modelling
}

\author{
Zhongyang Qiao ${ }^{1}$, Jibing Zhang ${ }^{1}$ and Yunzhu Gao ${ }^{2, *}$ \\ ${ }^{1}$ The Normal School of Beihua University, Jilin City, PR China \\ ${ }^{2}$ College of Mathematic and Statistics, Beihua University, Jilin City, PR China \\ ${ }^{*}$ Corresponding author
}

\begin{abstract}
In this paper, author studies of solution the energy for a nonlocal semilinear parabolic modeling. This modeling of equation that describes some phenomena of biological sciences is given. The author solves some problems involved in the model. First, the definition of energy is introduced. Secondly, the nonlocal parabolic equation with positive initial energy is considered. Finally, the existence and blow-up of initial energy of solution are given.
\end{abstract}

Keywords—positive initial energy; parabolic modeling; blowup

\section{INTRODUCTION}

In this paper, we consider the modeling of the positive initial energy to the following nonlocal parabolic equation.

$$
\begin{gathered}
\left\{\begin{array}{l}
w_{t}=\Delta w+|w|^{p-2} w-\frac{1}{|\Omega|} \int_{\Omega}|w|^{p-2} w d x, x \in \Omega, t>0, \\
\frac{\partial w}{\partial n}=0, \quad x \in \partial \Omega, t>0,
\end{array}\right. \\
\left\{\begin{array}{l}
w(x, 0)=w_{0}(x) \in C^{2, \alpha}(\bar{\Omega}), \quad x \in \Omega, \\
\int_{\Omega} w_{0}(x) d x=0, \quad \text { with } w_{0}(x) \text { not identical to zero, }
\end{array}\right.
\end{gathered}
$$

where, $\Omega$ is a bounded domain in $R^{N}(N \geq 1)$, with a smooth boundary $\partial \Omega$ and $p>2$.

There have been many literatures which consider properties of solutions to partial differential equations with a homogeneous Neumann boundary conditions. It is $\mathrm{s}$ immediately seen that the integral (or the mean value) of $w$ is conserved (at least once you make precise the meaning of the solution). The above model arises from nuclear science where the growth of temperature is known to be very fast, like $w^{p}$, but some absorption catalytic material is put into the system in such a way that the total mass is conserved. It can also be used to model other phenomena in population dynamics and biological sciences where the total mass is often conserved or known, but the growth of a certain cell is known to be of some form. Since mathematically we do not require that $w(x, t)$ is nonnegative, we use $|w|^{p-2} w$ instead of $w^{p}$ in our problem (see [1]).

One of the simplest examples of a nonlocal and quadratic equation is

$$
u_{t}=\Delta u+u^{2}-\frac{1}{|\Omega|} \int_{\Omega} u^{2} d x
$$

with a Neumann boundary condition such that the quantity $\int_{\Omega} u(x, t) d x$ is conserved. This equation is also related to Navier-Stokes equations on an infinite slab for other reasons explained in [2]. Wang and Wang [3] considered a more general problem of the form

$$
u_{t}-d \Delta u=\int_{\Omega} u^{q} d x-k u^{p}
$$

with Neumann or Dirichlet boundary conditions and positive initial data, where $d>0$ is a constant. They showed the global existence and exponential decay of the solutions when $p=q,|\Omega| \leq k$ and the boundary condition is of Neumann type. They also obtained a blow-up result under the assumption that the initial datum is bigger than some “Gaussian function”' in the case of $|\Omega|>k$.

In 1995, Hu and Yin [1] established the nonglobal existence result for problem (1) under the condition

$$
E(0)=\int_{\Omega}\left[\frac{1}{2}\left|\Delta u_{0}\right|^{2}-\frac{1}{p+1}\left|u_{0}\right|^{p+1}\right] d x \leq-C
$$

by using a convexity argument, where $C>0$ is a constant depending on the measure of $\Omega$.

EI Soufietal. in their work [4] investigated a slightly different problem: 


$$
u_{t}-\Delta u=|u|^{p}-\frac{1}{|\Omega|} \int_{\Omega}|u|^{p} d x
$$

with the same initial and boundary conditions as are given in Problem (1)-(2). They established a relationship between the finite time blow-up of solutions and the negativity of the initial energy for $1<p \leq 2$ and proposed a conjecture that the relationship might hold for all $p>1$, a positive answer to which was given by Jazar in [5].

In the works mentioned above, the initial energies are supposed to be negative or nonpositive to ensure the occurrence of the blow-up. To the best of our knowledge, there are fewer works in which positive initial energies guarantee blow-up, of which we refer to [6]. In this work, we improve the blow-up results obtained in [1, 4, 5] to Problem (1)-(2) in some sense and show that certain solutions with positive initial energy may also blow up in finite time. The existence and uniqueness of this problem for small time $t$ is clear, by the standard theory of parabolic estimates and the contraction mapping principle. It can also be obtained from the classical semigroup theory more or less directly. It is also clear that the solution can be extended in the t direction, as long as the $L^{\infty}$ norm of the solution remains finite. Moreover, the solution is a classical one; see [7].

Denote by $W(\Omega)$ the subspace of $W^{1,2}(\Omega)$, the element $w$ of which satisfies $\int_{\Omega} w d x=0$. We equip $W(\Omega)$ with the norm

$$
\|w\|=\left(\int_{\Omega}|\nabla w|^{2} d x\right)^{\frac{1}{2}} .
$$

By using the Poincaré inequality, we see that this norm is equivalent to the classical norm equipped with $W^{1,2}(\Omega)$. Let $\mathrm{B}$ be the optimal constant of the embedding inequality

$$
\|w\|_{p} \leq B\|\nabla w\|_{2}, \quad w \in W(\Omega)
$$

$$
\begin{gathered}
\alpha_{1}=B^{-\frac{p}{p-2}} \\
E_{1}=\left(\frac{1}{2}-\frac{1}{p}\right) B^{-\frac{2 p}{p-2}}>0
\end{gathered}
$$

and

$$
\begin{gathered}
E(t)=\int_{\Omega}\left[\frac{1}{2}|\nabla w|^{2}-\frac{1}{p}|w|^{p}\right] d x \\
\text { II. PRELIMINARY }
\end{gathered}
$$

We begin this section with the following lemma.

Lemma 2.1. $E(t)$ defined in (5) is nonincreasing about $t$.

Proof. Combining with integrating by parts and a direct computation, we have

$$
\begin{aligned}
\frac{d}{d t} E(t) & =-\int_{\Omega} w_{t}\left(\Delta w+|w|^{p-2} w\right) d x \\
& =-\int_{\Omega} w_{t}^{2} d x-\frac{1}{|\Omega|} \int_{\Omega}|w|^{p-2} w d x \int_{\Omega} w_{t} d x \\
& =-\int_{\Omega} w_{t}^{2} d x \leq 0
\end{aligned}
$$

Thus, $E(t)$ is nonincreasing in $t$.

Next, we first establish the following two lemmas by applying the idea of Vitillaro in [8]. A similar idea was used by Liu and Wang in [6]. The first lemma gives a lower bound estimate of the $L^{2}$ norm of the solution $w(x, t)$.

Lemma 2.2. Suppose that $w(x, t)$ is the solution of (1)(2). Assume that $E(0)<E_{1}$ and $\left\|\nabla w_{0}\right\|>\alpha_{2}$. Then there exists a positive constant $\alpha_{2}>\alpha_{1}$ such that

$$
\|\nabla w\|_{2} \geq \alpha_{2}, \forall t \geq 0
$$

and

$$
\|w\|_{p} \geq B \alpha_{2}, \forall t \geq 0
$$

Proof. We obtain from (3) and (5) that 


$$
\begin{aligned}
E(t) & \geq \frac{1}{2}\|\nabla w\|_{2}^{2}-\frac{1}{p} B^{p}\|\nabla w\|_{2}^{p} \\
& =\frac{1}{2} \alpha^{2}-\frac{1}{p} B^{p} \alpha^{p \stackrel{\Delta}{=}} h(\alpha)
\end{aligned}
$$

where, $\alpha=\|\nabla w\|_{2}$. It is easy to verify that $h$ is increasing for $0<\alpha<\alpha_{1}$, decreasing for $\alpha>\alpha_{1} ; h(\alpha) \rightarrow-\infty$ as $\alpha \rightarrow+\infty$ and $h\left(\alpha_{1}\right)=E_{1}$, where $\alpha_{1}$ and $E_{1}$ are given in (4). Since $E(0)<E_{1}$, there exists an $\alpha_{2}>\alpha_{1}$ such that $h\left(\alpha_{2}\right)=E(0)$. Let $\alpha_{0}=\left\|\nabla w_{0}\right\|_{2}$; then we have $h\left(\alpha_{0}\right) \leq E(0)=h\left(\alpha_{2}\right)$ by (8), which implies that $\alpha_{0}>\alpha_{2}$ since $\alpha_{0}, \alpha_{2} \geq \alpha_{1}$. To prove (6), we suppose on the contrary that $\left\|\nabla w\left(\cdot, t_{0}\right)\right\|_{2}<\alpha_{2}$ for some $t_{0}>0$. By the continuity of $\|\nabla w(\cdot, t)\|_{2}$, we may choose $t_{0}$ such that $\left\|\nabla w\left(\cdot, t_{0}\right)\right\|_{2}<\alpha_{1}$. Then it follows from (8) that $E(0)=h\left(\alpha_{2}\right)<h\left\|\nabla w\left(\cdot, t_{0}\right)\right\|_{2} \leq E\left(t_{0}\right)$, which contradicts Lemma 2.1. Hence (6) is proved.

To prove (7), we see from (5) that

$$
\frac{1}{2}\|\nabla w\|_{2}^{2} \leq E(0)+\frac{1}{p} \int_{\Omega}|w|^{p} d x
$$

which implies that

$$
\begin{gathered}
\frac{1}{p} \int_{\Omega}|w|^{p} d x \geq \frac{1}{2}\|\nabla w\|_{2}^{2}-E(0) \geq \frac{1}{2} \alpha_{2}^{2}-E(0) \\
=\frac{1}{2} \alpha_{2}^{2}-h\left(\alpha_{2}\right)=\frac{1}{p} B^{p} \alpha_{2}^{p} .
\end{gathered}
$$

Therefore, (7) holds. The proof is complete.

Let

$$
H(t)=E_{1}-E(t), \quad t>0
$$

Then we have:

Lemma 2.3. For all $t \geq 0$,

$$
0<H(0) \leq H(t) \leq \frac{1}{p} \int_{\Omega}|w|^{p} d x .
$$

Proof. By Lemma 2.1, we see that $H^{\prime}(t) \geq 0$. Thus $H(t) \geq H(0)>0, \quad t \geq 0$.
From (5) and (9) we see that $H(t)=E_{1}-\frac{1}{2}\|\nabla w\|_{2}^{2}+\frac{1}{p} \int_{\Omega}|w|^{p} d x$.

Combining (8) with (7) we have

$$
E_{1}-\frac{1}{2}\|\nabla w\|_{2}^{2} \leq E_{1}-\frac{1}{2} \alpha_{1}^{2}=-\frac{1}{p} B^{p} \alpha_{1}^{p} \leq 0, \quad t \geq 0
$$

which implies the conclusion of the lemma.

\section{MAIN RESULTS}

In this section, the blow-up of weak solutions will be studied. On the basis of the above lemmas, we are now in a position to prove the following Theorem:

Theorem 3.1 Let $2<p \leq+\infty$ when $N=1,2$, $2<p \leq \frac{2 N}{N-2}$ when $N \geq 3$ and let $w(x, t)$ be a classical solution of (1)-(2). Assume that the initial datum $w_{0}(x)$ is chosen to ensure that $E(0)<E_{1}$ and $\left\|\nabla w_{0}\right\|_{2}>\alpha_{1}$ hold. Then the solution $w(x, t)$ blows up in finite time.

Proof. Define $F(t)=\frac{1}{2} \int_{\Omega} w^{2}(x, t) d x$ and differentiate $F$ to get

$$
\begin{aligned}
F^{\prime}(t) & =\int_{\Omega} w w_{t} d x \\
& =\int_{\Omega} w\left(\Delta w+|w|^{p-2} w-\frac{1}{|\Omega|} \int_{\Omega}|w|^{p-2} w d x\right) d x \\
& =\int_{\Omega}|w|^{p} d x-\int_{\Omega}|\nabla w|^{2} d x \\
& =\int_{\Omega}|w|^{p} d x-2 E(t)-\frac{2}{p} \int_{\Omega}|w|^{p} d x \\
& =\frac{p-2}{p} \int_{\Omega}|w|^{p} d x-2 E_{1}+2 H(t)
\end{aligned}
$$

By using (4) and (7), we have

$$
\begin{aligned}
2 E_{1} & =2\left(\frac{1}{2}-\frac{1}{p}\right) B^{-\frac{2 p}{p-2}} \\
& =\frac{\alpha_{1}^{p}}{\alpha_{2}^{p}} \frac{p-2}{p} B^{p} \alpha_{2}^{p} \\
& \leq \frac{\alpha_{1}^{p}}{\alpha_{2}^{p}} \frac{p-2}{p} \int_{\Omega}|w|^{p} d x .
\end{aligned}
$$


Substituting the above inequality into (10), we obtain

$$
\begin{aligned}
F^{\prime}(t) & \geq\left(1-\frac{\alpha_{1}^{p}}{\alpha_{2}^{p}}\right) \frac{p-2}{p} \int_{\Omega}|w|^{p} d x+2 H(t) \\
& =C_{0} \int_{\Omega}|w|^{p} d x+2 H(t) \geq 0
\end{aligned}
$$

Where

$$
C_{0}=\left(1-\frac{\alpha_{1}^{p}}{\alpha_{2}^{p}}\right) \frac{p-2}{p}>0
$$

Next we estimate $F^{\frac{p}{2}}(t)$ by using Hölder's inequality:

$$
F^{\frac{p}{2}}(t)=\left(\frac{1}{2} \int_{\Omega} w^{2}(x, t) d x\right)^{\frac{p+1}{2}} \leq C \int_{\Omega}|w|^{p} d x
$$

where $C>0$ is a constant depending only on $|\Omega|$ and $p$. By combining (11) with (12) we have

$$
F^{\prime}(t) \geq \gamma F^{\frac{p}{2}}(t)
$$

where $\gamma=\frac{C_{0}}{C}>0$. A direct integration of (13) from 0 to $t$ yields

$$
F^{\frac{p-2}{2}}(t) \geq \frac{1}{F^{\frac{2-p}{2}}(0)-\frac{p-2}{2} \gamma t}
$$

Then $F(t)$ blows up at a finite time $T^{*} \leq \frac{F^{\frac{2-p}{2}}(0)}{\frac{p-2}{2} \gamma}$, and so does $w(x, t)$. The proof of Theorem 3.1 is complete.

\section{ACKNOWLEDGMENT}

The authors would like to thank the referees for their valuable comments on and suggestions regarding the original manuscript. The project was supported by Tianyuan FC of NSFC (11526035). Science and Technology Research Project of Jilin Provincial Department of Education (JJKH20170024KJ).

\section{REFERENCES}

[1] B. Hu, H. M. Yin, Semi-linear parabolic equations with prescribed energy, Rend. Circ. Math. Palermo 44(3) (1995) 479-505.

[2] C. Budd, B. Dold, A. Stuart, Blow-up in a system of partial differential equations with conserved first integral II. Problems with convection, SIAM J. Appl. Math. 54(3) (1994) 610-640.

[3] M. X. Wang, Y. M. Wang, Properties of positive solutions for non-local reaction-diffusion problems, Math. Methods Appl. Sci. 19(14) (1996) 1141-1156.

[4] Y. Gao, W. Gao, Existence and blow-up of solutions for a porous medium equation with nonlocal boundary condition, Applicable Analysis Vol. 90, No. 5, May 2011, 799-809.

[5] Raúl Ferreira, Blow-up for the non-local p-Laplacian equation with a reaction term, Nonlinear Analysis 75 (2012) 5499-5522

[6] W. J. Liu, M. X. Wang, Blow-up of solutions for a p-Laplacian equation with positive initial energy, Acta Appl. Math. 103(2) (2008) 141-146.

[7] A. Friedman, Partial Differential Equations of Parabolic Type, Prentice Hall, Englewood Cliffs, NJ, 1964.

[8] E. Vitillaro, Global nonexistence theorems for a class of evolution equations with dissipation, Arch. Ration. Mech. Anal. 149(2) (1999) 155-182.66. (2001) 